\title{
Osteopontin mediates obesity-induced adipose tissue macrophage infiltration and insulin resistance in mice
}

\author{
Takashi Nomiyama, ${ }^{1}$ Diego Perez-Tilve,, ${ }^{2}$ Daisuke Ogawa, ${ }^{1}$ Florence Gizard, ${ }^{1}$ Yue Zhao, ${ }^{1}$ \\ Elizabeth B. Heywood, ${ }^{1}$ Karrie L. Jones, ${ }^{1}$ Ryuzo Kawamori, ${ }^{3}$ Lisa A. Cassis, ${ }^{4}$ \\ Matthias H. Tschöp, ${ }^{2}$ and Dennis Bruemmer ${ }^{1,4}$
}

\begin{abstract}
${ }^{1}$ Division of Endocrinology and Molecular Medicine, University of Kentucky College of Medicine, Lexington, Kentucky, USA. ${ }^{2}$ Obesity Research Center, Department of Psychiatry, Genome Research Institute, University of Cincinnati, Cincinnati, Ohio, USA. ${ }^{3}$ Department of Medicine, Metabolism and Endocrinology, Juntendo University School of Medicine, Tokyo, Japan. ${ }^{4}$ Graduate Center for Nutritional Sciences, University of Kentucky, Lexington, Kentucky, USA.
\end{abstract}

\begin{abstract}
Obesity is associated with a state of chronic, low-grade inflammation characterized by abnormal cytokine production and macrophage infiltration into adipose tissue, which may contribute to the development of insulin resistance. During immune responses, tissue infiltration by macrophages is dependent on the expression of osteopontin, an extracellular matrix protein and proinflammatory cytokine that promotes monocyte chemotaxis and cell motility. In the present study, we used a murine model of diet-induced obesity to examine the role of osteopontin in the accumulation of adipose tissue macrophages and the development of insulin resistance during obesity. Mice exposed to a high-fat diet exhibited increased plasma osteopontin levels, with elevated expression in macrophages recruited into adipose tissue. Obese mice lacking osteopontin displayed improved insulin sensitivity in the absence of an effect on diet-induced obesity, body composition, or energy expenditure. These mice further demonstrated decreased macrophage infiltration into adipose tissue, which may reflect both impaired macrophage motility and attenuated monocyte recruitment by stromal vascular cells. Finally, obese osteopontin-deficient mice exhibited decreased markers of inflammation, both in adipose tissue and systemically. Taken together, these results suggest that osteopontin may play a key role in linking obesity to the development of insulin resistance by promoting inflammation and the accumulation of macrophages in adipose tissue.
\end{abstract}

\section{Introduction}

Obesity and the associated metabolic pathologies are the most common risk factors for type 2 diabetes and subsequent cardiovascular disease (1). Unequivocal evidence has demonstrated that obesity is associated with a state of chronic, low-grade inflammation characterized by abnormal cytokine production and activation of inflammatory signaling pathways in adipose tissue (2). Recent studies indicated that adipose tissue macrophages (ATMs) accumulating during diet-induced obesity (DIO) are not only an important source of adipose tissue inflammation but also alter insulin sensitivity in adipocytes: Weisberg et al. identified macrophage accumulation in obese adipose tissue and suggested that these macrophages are derived from the circulation (3). A critical observation made by Xu et al. further characterized these macrophages as an important mediator of insulin resistance (4). Collectively, these studies support the concept that ATMs infiltrating into obese adipose tissue from the circulation are a key source of inflammation in obesity and provide a causal link between obesity and the development of adipose tissue insulin resistance (5).

Nonstandard abbreviations used: AF, adipocyte fraction; ATM, adipose tissue macrophage; CCR2, C-C motif chemokine receptor 2; DIO, diet-induced obesity; EWAT, epididymal white adipose tissue; HFD, high-fat diet; LFD, low-fat diet; MCP-1, monocyte chemoattractant protein 1; OPN, osteopontin; PAI-1, plasminogen activator inhibitor 1 ; RQ, respiratory quotient; SVF, stromal vascular fraction.

Conflict of interest: The authors have declared that no conflict of interest exists. Citation for this article: J. Clin. Invest. 117:2877-2888 (2007). doi:10.1172/JCI31986.
The observation that macrophages infiltrate adipose tissue from the circulation has focused attention on the mechanisms by which these cells are recruited into obese adipose tissue (3). Accumulating evidence over the last decade has demonstrated that macrophage recruitment during inflammatory processes is dependent on the expression of osteopontin (OPN) (6-9). OPN is a secreted matrix glycoprotein and proinflammatory cytokine that has previously been characterized as a major component of cell-mediated immunity (8). Its ability to interact with integrin surface receptors through an Arg-Gly-Asp (RGD) sequence and with the CD44 receptor has established OPN as an important attachment and signaling molecule $(10,11)$. In bone tissue, for example, OPN facilitates the attachment of osteoclasts to the matrix (12). In addition to its role in bone metabolism, a variety of studies have provided evidence that OPN is highly secreted by macrophages at sites of inflammation where it mediates monocyte adhesion (12), migration (7), differentiation (13), and phagocytosis (14). Using OPN-deficient mice crossed to atherosclerosis-prone apo $\mathrm{E}^{-/-}$mice, we (9) and others (15) have further recently demonstrated that OPN deficiency attenuates the development of atherosclerosis. In these studies OPN expression was found to be essential for monocyte motility and inflammatory gene expression; in particular, OPN deficiency in macrophages decreased the development of atherosclerosis (9). It is now well recognized that OPN induces chemotaxis of monocytes and promotes cellular motility via direct interaction with its receptors $(10,11)$. 

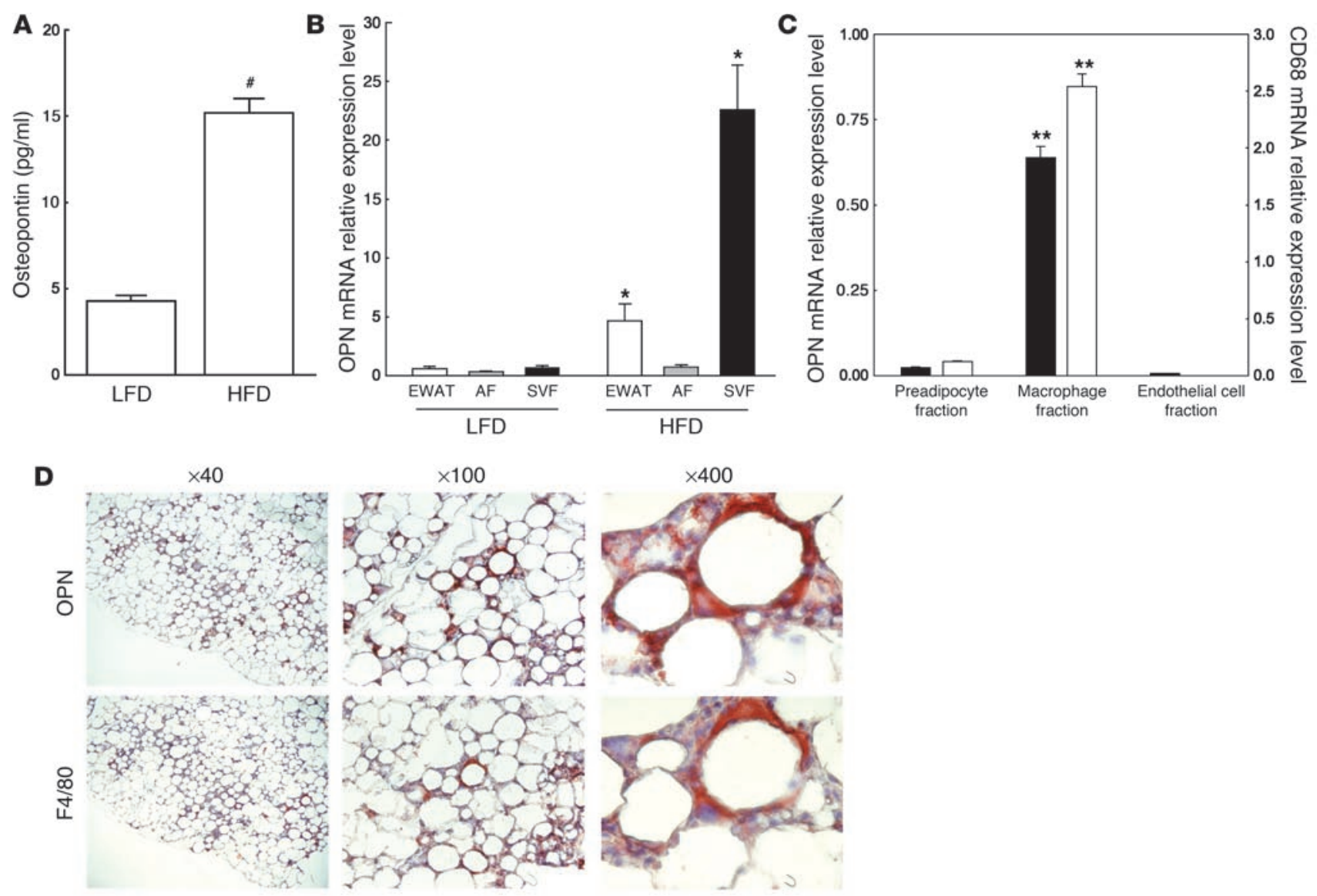

\section{Figure 1}

OPN plasma levels in DIO and expression of OPN in adipose tissue. C57BL/6 mice ( $n=10 /$ group) were fed a LFD or HFD for 20 weeks. (A) OPN plasma levels were analyzed by ELISA and data expressed as mean \pm SEM. ${ }^{\prime} P<0.005$. (B) Adipose tissues were isolated and OPN mRNA expression was analyzed in whole EWAT, the AF, and the SVF. Data are presented as relative OPN mRNA expression normalized to TFIIB mRNA expression and are expressed as mean \pm SEM. ${ }^{*} P<0.05$, compared with LFD. (C) SVFs isolated from EWATs of obese wild-type mice $(n=6)$ were pooled and separated into macrophages, endothelial cells, and preadipocytes using magnetic immunoaffinity isolation. Cell fractions were analyzed for OPN (black bars, left $y$ axis) and CD68 (white bars, right $y$ axis) mRNA expression. Data are presented as mRNA expression relative to TFIIB mRNA expression and are expressed as mean \pm SEM. ${ }^{\star *} P<0.01$ compared with preadipocyte or endothelial cell fraction. (D) Paraffin-embedded EWAT was analyzed for OPN immunoreactivity and F4/80-positive macrophage content. Sections were counterstained with hematoxylin and images obtained at the indicated magnifications.

Based on the evidence that obesity is associated with infiltration (3) and activation (16) of macrophages in adipose tissue combined with recent studies characterizing OPN as an important component of cell-mediated immune responses and monocyte motility $(10,11)$, we investigated the expression of OPN in adipose tissue and examined the role of OPN for macrophage accumulation in adipose tissue. Since macrophages and associated proinflammatory cytokines contribute to adipose tissue insulin resistance during DIO (4), we further analyzed the effect of OPN deficiency on systemic insulin resistance. Using a murine model of DIO, we report that OPN secretion is increased during obesity and highly expressed in ATMs, characterizing OPN as a cytokine secreted by adipose tissue. OPN deficiency attenuated ATM content, adipose tissue, and systemic inflammation and improved insulin resistance. These studies outline a previously unrecognized role for OPN in mediating ATM recruitment and identify OPN as an important link between adipose tissue-derived inflammatory processes and insulin resistance.

\section{Results}

OPN expression in adipose tissue increases during DIO. To analyze whether OPN plasma levels change during DIO, C57BL/6 wildtype mice ( $n=10$ /group) were maintained either on a low-fat diet (LFD; $10 \% \mathrm{kcal}$ from fat) or high-fat diet (HFD; 60\% kcal from fat) for 20 weeks. Compared with the LFD group, mice fed a HFD gained $26.1 \mathrm{~g}$ after a 20 -week feeding period $(28.2 \pm 4.1$ versus $54.3 \pm 5.3 \mathrm{~g} ; P<0.005)$. As depicted in Figure 1A, OPN plasma levels analyzed by ELISA were significantly elevated in obese mice fed a HFD compared with the lean control mice fed a LFD $(15.2 \pm 0.8$ versus $4.3 \pm 0.3 \mathrm{pg} / \mathrm{ml}$, respectively; $P<0.005$ ).

Epididymal white adipose tissue (EWAT) from these mice was next analyzed for OPN mRNA expression to examine whether the OPN transcript is expressed in adipose tissue and whether this is modulated by the obese phenotype. In mice fed a LFD, OPN mRNA expression levels were negligible in whole adipose tissues as well in fractionated tissues (Figure 1B). However, feeding a HFD resulted in a significant 8.1-fold $(P<0.01)$ increase of OPN transcript levels in 

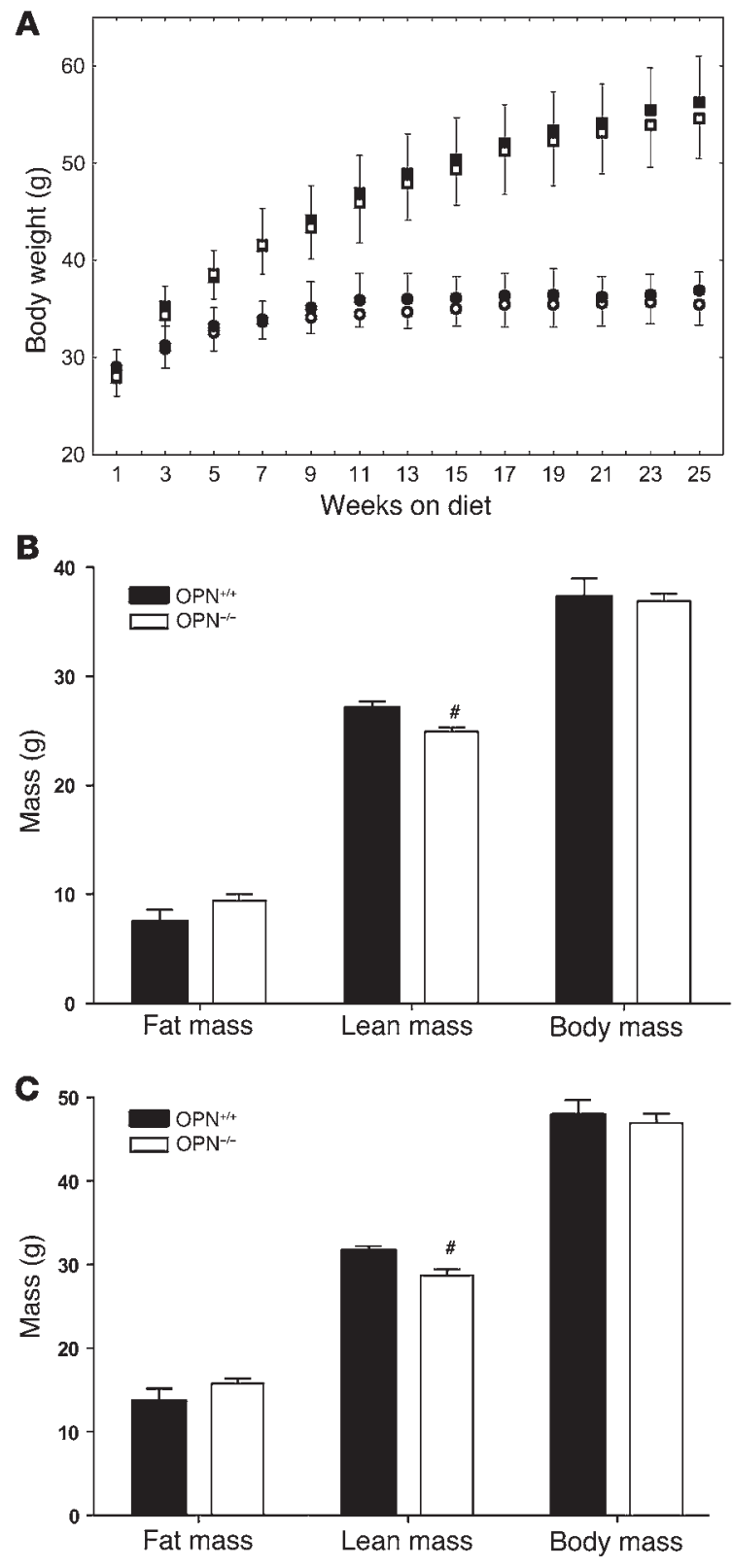

whole adipose tissues. Separation of the adipose tissue demonstrated that there was a profound 35.9-fold $(P<0.001)$ increase of OPN mRNA in the stromal vascular fraction (SVF). In contrast, there was an insignificant 2.2-fold increase $(P=0.091)$ of OPN in the adipocyte fraction $(\mathrm{AF})$, indicating that the increase of OPN mRNA in whole adipose tissue was primarily due to an increase of transcript levels in the SVF. The observation that OPN mRNA expression in the AF increased only modestly during DIO prompted us to corroborate these findings in vitro and to analyze the regulation of OPN during the differentiation of 3T3-L1 preadipocytes. Use of this model system revealed that OPN mRNA levels decrease during the differentiation process (Supplemental Figure 1; supplemental material available online with this article; doi:10.1172/JCI31986DS1). Previous studies have indicated that PPAR $\gamma$ activation suppresses OPN transcription in macrophages (17). Since PPAR $\gamma$ expression is induced during adipocyte differentiation, we analyzed the transcrip-

\section{Figure 2}

$\mathrm{DIO}$ and body composition in wild-type $\mathrm{OPN}^{+/+}$and $O P N^{-/-}$mice. (A) Wild-type OPN ${ }^{+/+}$(black symbols, $n=12$ ) and $\mathrm{OPN}^{-/-}$(white symbols, $n=12$ ) mice were fed a LFD (circles) or HFD (squares). Weight gain was followed for 25 weeks and data expressed as mean \pm SEM. Body composition (fat mass, lean mass, and body mass) before (B) and after (C) feeding a HFD ( $n=7-8$ /group) was analyzed in $\mathrm{OPN}^{+/+}$(black bars) and $\mathrm{OPN}^{-1-}$ (white bars) mice by quantitative NMR. Data are presented as mean \pm SEM. ${ }^{\#} P<0.05$, compared with $O P N^{+/+}$mice.

tional regulation of a $2-\mathrm{kb}$ OPN promoter construct by PPAR $\gamma$ in 3T3-L1 preadipocytes. Interestingly, both ligand-induced activation of PPAR $\gamma$ and overexpression of a constitutively active PPAR $\gamma$ suppressed OPN promoter activity (Supplemental Figure 2), providing a potential mechanism for the observed decrease of OPN transcript levels during adipocyte differentiation.

Given the high expression of OPN mRNA in the SVF, we next characterized the cell types expressing OPN within the SVF using magnetic immunoaffinity isolation of ATMs, endothelial cells, and preadipocytes. Analysis of expression of CD68 mRNA encoding a $110-\mathrm{kDa}$ transmembrane glycoprotein that is highly expressed by monocytes and tissue macrophages (18) confirmed that $95.1 \%$ of the CD68 mRNA in the SVF was found in the macrophage fraction (Figure 1C). Using this approach, OPN mRNA expression in the SVF was primarily observed in macrophage isolates, while considerably less OPN mRNA was detected in the preadipocyte fraction and in the endothelial cell fraction.

To confirm that OPN is expressed by ATMs, we performed immunohistochemistry on epididymal fat pads isolated from obese C57BL/6 mice fed a HFD for 3 months. As the majority of studies analyzing ATM content in murine models previously employed an antibody raised against the F4/80 antigen $(3,19)$, a marker specific for mature macrophages (20), we stained consecutive sections for F4/80 and OPN. Negative controls included lack of chromogen development following incubation with the same concentrations of control nonimmune IgG in the presence of the secondary antibody or incubation with the primary antibody in absence of the secondary antibody (data not shown). As reported recently (3), DIO was associated with the accumulation of macrophages that were frequently observed in obese adipose tissue forming crown-like aggregates surrounding adipocytes (Figure 1D). Staining for OPN revealed a striking similarity with F4/80 immunoreactivity, and higher magnification of serial sections confirmed OPN expression in F4/80 positive macrophages. In concert, these results suggest that OPN plasma levels increase during obesity and that during DIO OPN mRNA is primarily expressed by ATMs accumulating in adipose tissue.

OPN deficiency does not affect DIO. To further determine the role of OPN for the development of DIO, adipose tissue inflammation, and insulin resistance, we next fed male $O P N^{-/-}$and littermate wild-type $O P N^{+/+}$mice ( $n=12 /$ group) either a LFD or a HFD for 25 weeks. Consistent with our previous studies (9), there were no differences in weights between $O P N^{-/-}$and $\mathrm{OPN}^{+/+}$mice at baseline or after feeding a LFD for 25 weeks. In mice fed a HFD for 25 weeks, there was a statistically nonsignificant trend toward lower body mass in the $\mathrm{OPN}^{-/-}$mice compared with $\mathrm{OPN}^{+/+}$wild-type mice $(54.6 \pm 4.1$ versus $56.2 \pm 4.8 \mathrm{~g}$, respectively; $P=0.087$; Figure $2 \mathrm{~A})$. To examine whether there are differences in lean or fat mass, we analyzed body composition in $O P N^{-/-}$and $O P N^{+/+}$mice using NMR before (Figure 2B) and after (Figure 2C) feeding a HFD. This approach revealed no difference in fat mass between lean or obese 


\section{Table 1}

Food intake, energy expenditure, $\mathrm{RQ}$, and locomotor activity of animals in this study

\begin{tabular}{|c|c|c|c|c|c|}
\hline \multirow[t]{2}{*}{ Parameter } & \multirow[t]{2}{*}{ Cycle } & \multicolumn{2}{|c|}{ Before HFD } & \multicolumn{2}{|c|}{ After HFD } \\
\hline & & $O P N+/+$ & $O P N-/$ & $O P N^{+/+}$ & OPN-/- \\
\hline Food intake (g) & $\begin{array}{l}\text { Total } \\
\text { Light } \\
\text { Dark }\end{array}$ & $\begin{array}{r}10.2 \pm 0.7 \\
3.9 \pm 0.6 \\
6.2 \pm 0.2\end{array}$ & $\begin{array}{l}9.7 \pm 1.1 \\
4.2 \pm 0.7 \\
5.5 \pm 0.8\end{array}$ & $\begin{array}{l}5.7 \pm 0.8 \\
1.5 \pm 0.3 \\
4.2 \pm 0.8\end{array}$ & $\begin{array}{l}6.8 \pm 0.7 \\
2.9 \pm 0.5^{A} \\
3.8 \pm 0.3\end{array}$ \\
\hline Energy expenditure (kcal/kg) & $\begin{array}{l}\text { Total } \\
\text { Light } \\
\text { Dark }\end{array}$ & $\begin{array}{l}711.5 \pm 17.5 \\
334.1 \pm 12.2 \\
393.4 \pm 7.4\end{array}$ & $\begin{array}{l}733.8 \pm 22.5 \\
346.9 \pm 11.2 \\
402.6 \pm 12.5\end{array}$ & $\begin{array}{l}770.3 \pm 16.8 \\
367.3 \pm 8.1 \\
419.3 \pm 10.7\end{array}$ & $\begin{array}{l}847.4 \pm 26.4 \\
419.2 \pm 11.4 \\
444.6 \pm 16.2\end{array}$ \\
\hline $\mathrm{RQ}\left(\mathrm{vCO}_{2} / \mathrm{vO}_{2}\right)$ & $\begin{array}{l}\text { Total } \\
\text { Light } \\
\text { Dark }\end{array}$ & $\begin{array}{l}0.862 \pm 0.010 \\
0.827 \pm 0.016 \\
0.900 \pm 0.015\end{array}$ & $\begin{array}{l}0.876 \pm 0.016 \\
0.836 \pm 0.016 \\
0.909 \pm 0.016\end{array}$ & $\begin{array}{l}0.773 \pm 0.011 \\
0.745 \pm 0.016 \\
0.856 \pm 0.010\end{array}$ & $\begin{array}{l}0.796 \pm 0.011 \\
0.815 \pm 0.013^{A} \\
0.847 \pm 0.011\end{array}$ \\
\hline $\begin{array}{l}\text { Locomotor activity } \\
\text { (light beam breaks) }\end{array}$ & $\begin{array}{l}\text { Total } \\
\text { Light } \\
\text { Dark }\end{array}$ & $\begin{array}{l}34,857.1 \pm 1,996.1 \\
11,994.7 \pm 422.1 \\
22,862.4 \pm 1,892.1\end{array}$ & $\begin{array}{l}28,367.1 \pm 2,268.5 \\
11,713.5 \pm 1,317.2 \\
16,653.6 \pm 1,561.7\end{array}$ & $\begin{array}{l}32,993.4 \pm 3,230.1 \\
10,164.1 \pm 632.2 \\
21,149.0 \pm 2,446.6\end{array}$ & $\begin{aligned} 24,489.4 & \pm 2,748.5 \\
6,409.6 & \pm 568.1 \\
17,461.8 & \pm 1,983.1\end{aligned}$ \\
\hline
\end{tabular}

$O P N^{+/+}$wild-type and $O P N^{-/-}$mice ( $n=7-8 /$ group) were analyzed before (at 8 weeks of age, while on standard chow diet) and after feeding a HFD. Data is expressed as mean \pm SEM. ${ }^{A} P<0.05, O P N^{-1-}$ compared with $O P N^{+/+}$.

$O P N^{-/-}$and $O P N^{+/+}$mice. However, compared with wild-type mice, there was a modest but significant decrease in lean body mass of $\mathrm{OPN}^{-/-}$mice in the absence of a difference in whole body mass. Therefore, OPN deficiency had no effect on the development of DIO in mice fed a HFD.

We next analyzed whether OPN deficiency affects energy metabolism by recording cumulative measurements for food intake, energy expenditure, respiratory quotient (RQ), and locomotor activity (Table 1). Initial recordings were obtained at 8 weeks of age on mice fed a standard chow diet, followed by measurements after feeding $\mathrm{OPN}^{-/-}$and wild-type $\mathrm{OPN}^{+/+}$a HFD for 20 weeks. Consistent with the observed lack of any overt differences in whole body mass and adiposity, no statistically significant differences in total food intake, energy expenditure, RQ, or locomotor activity were observed. However, $O P N^{-/-}$mice showed a modestly increased RQ during the 12-hour light cycle, indicating slightly higher carbohydrate utilization on HFD. This effect was likely a consequence of increased light phase food intake in the $\mathrm{OPN}^{-/-}$mice fed a HFD, as both increased RQ and food intake disappeared when analyzed over the entire 24-hour period.

OPN deficiency improves insulin sensitivity. HFD feeding and DIO in mice is associated with the development of insulin resistance (21), and to further explore whether insulin resistance is affected by OPN deficiency, we analyzed metabolic parameters in $\mathrm{OPN}^{-/-}$and wild-type $O P N^{+/+}$mice. No significant differences were observed in fasting blood glucose or plasma insulin concentrations between $O P N^{-/-}$and $\mathrm{OPN}^{+/+}$mice fed a LFD for 25 weeks (Table 2). Both plasma glucose and insulin levels significantly increased in obese wild-type mice fed a HFD. In contrast, in $O P N^{-1-}$ mice fed a HFD, plasma glucose levels were normal and insulin levels were significantly lower compared with the obese $\mathrm{OPN}^{+/+}$wild-type mice fed a HFD. Furthermore, HFD feeding in wild-type $\mathrm{OPN}^{+/+}$mice resulted in a significant increase in triglyceride levels, which was normalized in $\mathrm{OPN}^{-/-}$mice.

To further confirm that obese $O P N^{-/-}$mice are more insulin sensitive, we next analyzed glucose clearance following an intraperitoneal injection of insulin as a measure of insulin sensitivity. In these experiments, the insulin response in mice fed a LFD was not significantly affected by the genotype, although $O P N^{-/-}$ mice tended to be slightly more insulin sensitive. In obese $\mathrm{OPN}^{+/+}$ mice fed a HFD, glucose levels decreased by $16.4 \% 60$ minutes after insulin injection, whereas levels decreased by $49.3 \%$ in $\mathrm{OPN}^{-/-}$mice, indicating that the absence of OPN improved insulin sensitivity in obese mice (Figure 3A). The maximal insulin response after 60 minutes in obese $\mathrm{OPN}^{-/-}$mice fed a HFD reached levels observed in mice fed a LFD. Similarly, intraperitoneally injected glucose during an intraperitoneal glucose tolerance test was cleared faster and more effectively in obese $\mathrm{OPN}^{-/-}$mice compared with $\mathrm{OPN}^{+/+}$(Figure $3 \mathrm{~B}$ ). In concert, these data confirm that insulin sensitivity is increased in obese $\mathrm{OPN}^{-/-}$compared with wild-type $\mathrm{OPN}^{+/+}$mice.

\section{Table 2}

Metabolic parameters of mice examined in this study

\begin{tabular}{|c|c|c|c|c|c|c|c|c|}
\hline \multirow[t]{2}{*}{ Parameter } & \multicolumn{2}{|c|}{ LFD } & \multirow{2}{*}{$\begin{array}{c}P \\
O P N^{+/+} \text {VS. } \\
O P N^{-/-}\end{array}$} & \multicolumn{2}{|c|}{ HFD } & \multirow{2}{*}{$\begin{array}{c}P \\
O P N^{+/+} \text {vS. } \\
O P N^{-/-}\end{array}$} & \multirow{2}{*}{$\begin{array}{c}P \\
\text { OPN+/+ LFD } \\
\text { vs. HFD }\end{array}$} & \multirow{2}{*}{$\begin{array}{c}P \\
\text { OPN-/- LFD }^{-} \\
\text {vs. HFD }\end{array}$} \\
\hline & $\mathrm{OPN}^{+/+}$ & $O P N^{-/}$ & & $\mathrm{OPN}^{+/+}$ & $O P N^{-1}$ & & & \\
\hline Cholesterol (mg/dl) & $183.3 \pm 11.7$ & $183.4 \pm 7.2$ & 0.342 & $145.3 \pm 16.2$ & $166.1 \pm 11.1$ & 0.751 & 0.342 & 0.227 \\
\hline Triglycerides (mg/dl) & $55.1 \pm 5.4$ & $77.9 \pm 14.5$ & 0.751 & $112.9 \pm 7.9$ & $57.8 \pm 5.6$ & 0.009 & 0.026 & 0.227 \\
\hline Glucose (mg/dl) & $94.4 \pm 4.7$ & $101.8 \pm 9.3$ & 0.292 & $113.0 \pm 4.3$ & $99.7 \pm 5.6$ & 0.044 & 0.033 & 0.683 \\
\hline Insulin (pg/ml) & $159.1 \pm 39.1$ & $183.2 \pm 37.1$ & 0.541 & $1,976.4 \pm 425.5$ & $609.6 \pm 105.9$ & 0.041 & 0.023 & 0.046 \\
\hline
\end{tabular}



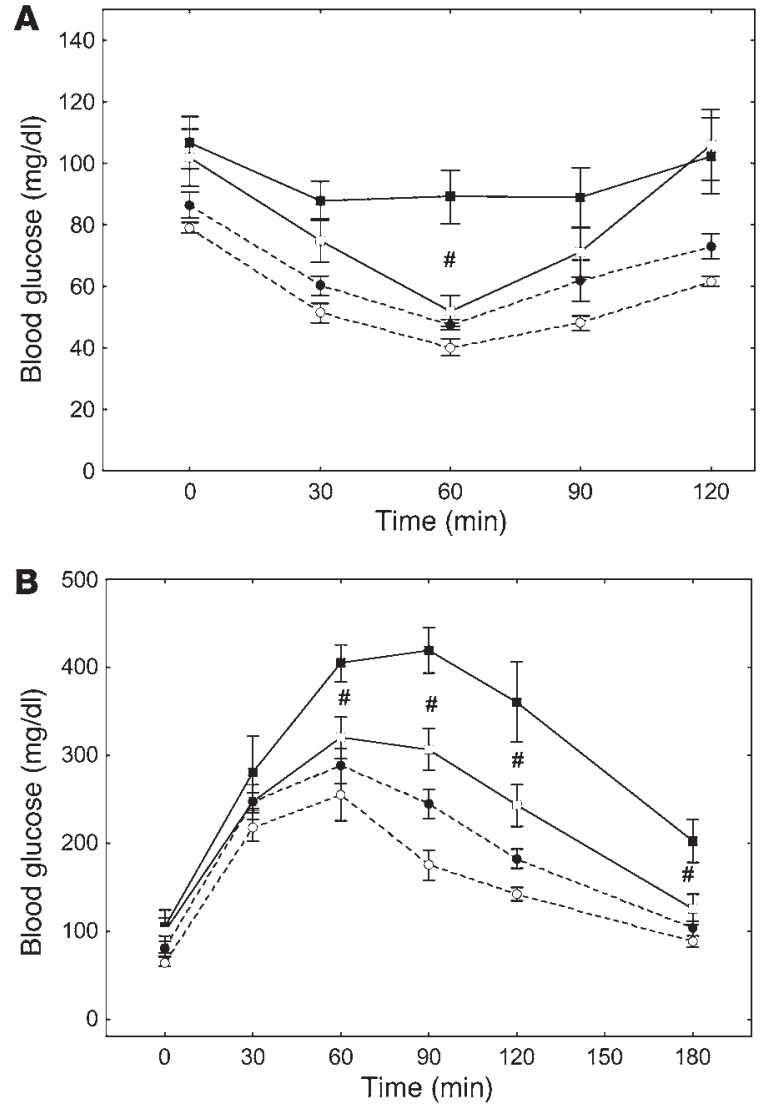

OPN deficiency decreases macrophage accumulation in obese adipose tissue. It has previously been demonstrated that ATMs accumulate during DIO, resulting in obesity-associated insulin resistance $(3,4)$. The evidence that OPN mediates the recruitment of macrophages in tissues (6-9) prompted us to further investigate the cause of increased insulin sensitivity in obese $O P N^{-/-}$mice and to determine whether OPN-deficient mice were protected from adipose tissue inflammation during obesity. We first analyzed macrophage content in epididymal adipose tissue pads isolated from $\mathrm{OPN}^{-/-}$and $\mathrm{OPN}^{+/+}$mice fed a LFD and HFD by immunohistochemical analysis. In epididymal adipose tissues no overt differences in adipocyte size or numbers between genders were noted. Using an absorbed rabbit antimouse macrophage antiserum, we observed a profound increase in ATM number in obese $\mathrm{OPN}^{+/+}$wild-type mice fed a HFD (Figure 4A). Typically, immunohistochemistry localized macrophages in crownlike clusters surrounding adipocytes. Despite having a similar fat mass following HFD feeding, obese epididymal adipose tissues isolated from OPN-deficient mice accumulated strikingly fewer ATMs (Figure 4A, lower panel). As the majority of studies analyzing ATM content in murine models have used an antibody against the F4/80 antigen $(3,19)$, we further sought to corroborate these observations using staining for F4/80. As depicted in Figure 4B, the majority of cells surrounding adipocytes expressed F4/80, and this immunoreactivity correlated with the staining obtained using the rabbit anti-mouse macrophage antiserum. When F4/80-positive ATMs were further quantified as a percentage of total cell number (Figure 4C), epididymal adipose tissue from obese $O P N^{-/}$mice fed a HFD contained fewer macrophages compared with wild-type $\mathrm{OPN}^{+/+}$mice $(19.1 \% \pm 2.0 \%$ versus $36.8 \% \pm 3.1 \% ; P<0.005)$.

\section{Figure 3}

Insulin sensitivity in $\mathrm{OPN}^{+/+}$and $O P N^{-/-}$mice. $O P N^{+/+}$(black symbols) and $O P N^{-1-}$ (white symbols) mice were fed a LFD (dashed lines) or HFD (solid lines) for 25 weeks. (A) Insulin sensitivity in these mice ( $n=6$ /group) was determined at the end of the feeding period following an intraperitoneal injection of insulin (1 U/kg body weight). (B) Glucose clearance ( $n=6 /$ group) was analyzed following an intraperitoneal challenge of $1 \mathrm{~g} / \mathrm{kg}$ body weight glucose. Data are presented as mean blood glucose concentration \pm SEM. ${ }^{\#} P<0.05, O P N^{-/}$compared with $O P N^{+/+}$mice fed HFD.

To further confirm this observation we next analyzed the macrophage content in whole and fractionated epididymal adipose tissue by quantitative real-time RT-PCR for CD68. As expected, CD68 mRNA increased during DIO in whole adipose isolated from $\mathrm{OPN}^{+/+}$mice (Figure 4D). High CD68 mRNA expression was detected in the SVF while transcript levels were negligible in the AF of both genotypes. Consistent with the data obtained by immunohistochemistry, CD68 mRNA transcript levels were significantly decreased in both whole adipose tissue and the SVF isolated from obese $\mathrm{OPN}^{-/-}$mice compared with wild-type $\mathrm{OPN}^{+/+}$ mice. In concert, immunohistochemistry and quantitative RT-PCR indicate that OPN deficiency prevents ATM accumulation during $\mathrm{DIO}$, although macrophage content is not completely rescued to the level of lean mice fed a LFD.

OPN mediates chemotaxis and amplifies monocyte chemoattractant protein 1-induced macrophage migration. OPN promotes cell motility and chemoattraction $(6,7,9,22)$, and so we next analyzed the effect of OPN deficiency on macrophage chemotaxis. Wild-type macrophage migration increased by $1.67 \pm 0.21$-fold $(P<0.05)$ when OPN was provided as substrate (Figure $5 \mathrm{~A}$ ). When cells were exposed to monocyte chemoattractant protein 1 (MCP-1), transwell migration of wild-type macrophages increased by $1.77 \pm 0.12$-fold $(P<0.05)$. MCP-1-induced chemotaxis was further increased by $2.41 \pm 0.28$-fold in the presence of OPN $(P<0.01)$. In comparison, OPN-deficient macrophages were hypomotile, and basal migration was only $62.6 \% \pm 2.26 \%$ that of the wild-type cells $(P<0.05)$. Although $O P N^{-/}$macrophages migrated by $1.94 \pm 0.12$-fold $(P<0.05)$ when recombinant OPN was provided, exogenous OPN only partially restored the wild-type phenotype. Furthermore, OPN-deficient macrophages failed to respond to MCP-1, which was only modestly restored in the presence of extracellular OPN $(2.54 \pm 0.32$-fold increase versus baseline; $P<0.01)$. We further sought to confirm these observations ex vivo and analyzed migration of macrophages wild-type for OPN toward stromal vascular cells isolated from $\mathrm{OPN}^{+/+}$and $O P N^{-/-}$mice fed a LFD or HFD for 25 weeks. As depicted in Figure 5B, macrophage migration to stromal vascular cells isolated from obese wild-type mice was significantly increased $3.1 \pm 0.6$-fold relative to migration toward cells isolated from lean mice $(P<0.05)$. Compared with the migratory response elicited by stromal vascular cells of obese wild-type mice, transwell macrophage migration was significantly decreased to $53.3 \% \pm 3.2 \%$ when macrophages migrated toward stromal vascular cells isolated from obese $\mathrm{OPN}^{-/-}$mice $(P<0.05)$. In concert, these experiments indicate that OPN amplifies MCP-1-directed macrophage chemotaxis and that OPN deficiency in stromal vascular cells alters obesity-induced macrophage recruitment.

$\mathrm{OPN}^{-/-}$mice are protected from obesity-associated adipose tissue and systemic inflammation. Since accumulating evidence outlines a key role of DIO in the development of low-grade systemic inflammation 
A

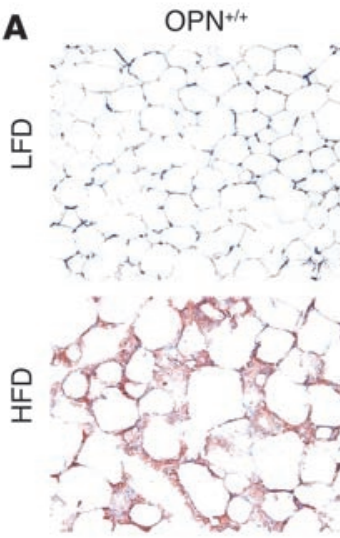

B

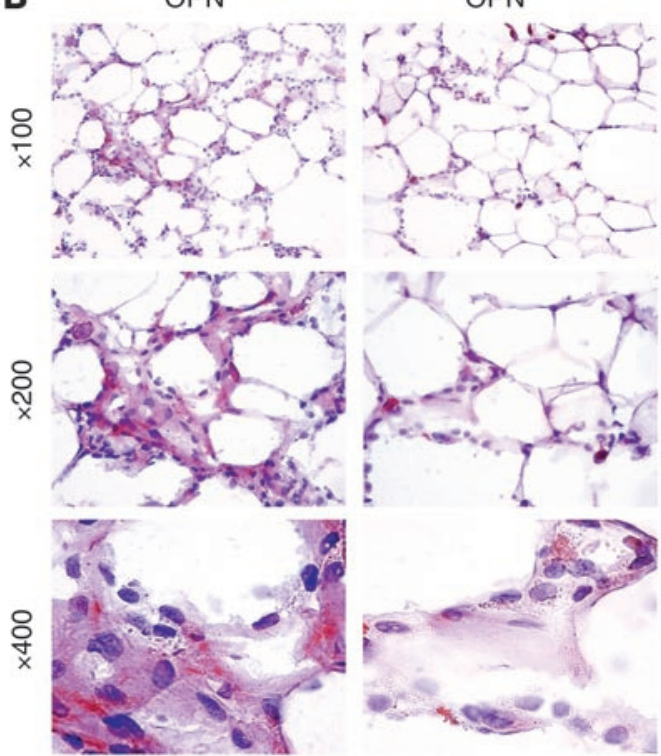

C

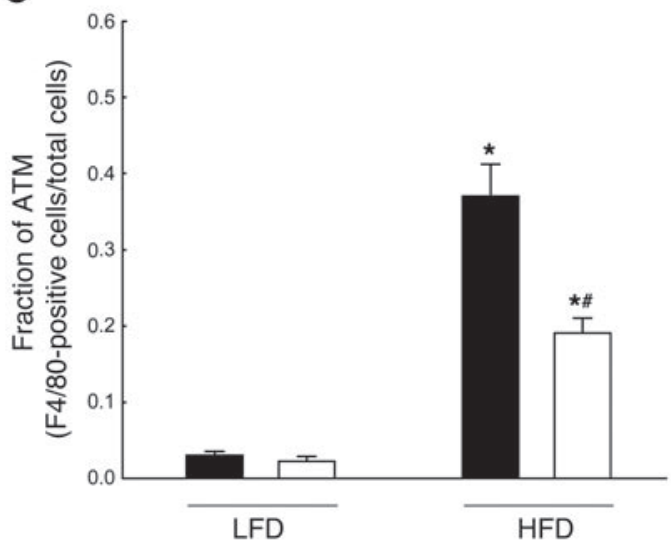

D

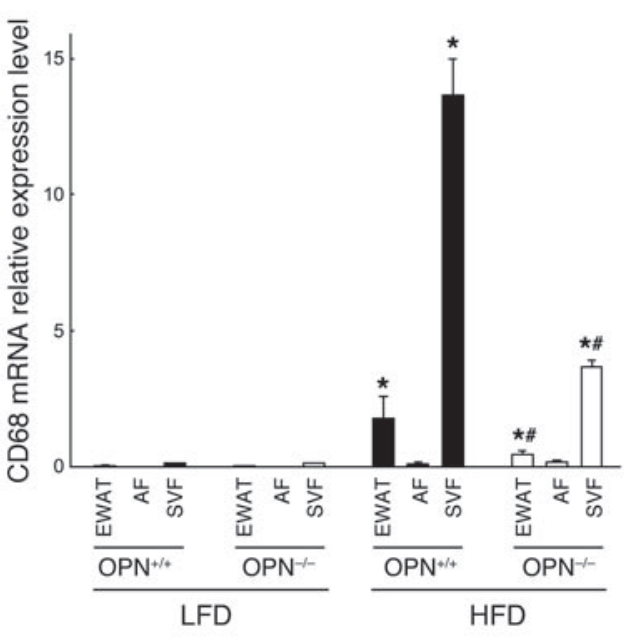

\section{Figure 4}

OPN deficiency decreases ATM content in obese mice. (A) ATM content was determined by immunohistochemical analysis of epididymal adipose tissues isolated from $O P N^{+/+}$and $O P N^{-/-}$mice fed a LFD or HFD. Adipose tissues were stained using an absorbed rabbit anti-mouse macrophage antiserum (original magnification, $\times 100$ ). (B) Epididymal adipose tissues from obese $O P N^{+/+}$and $O P N^{-/}$mice were analyzed for macrophage content using an F4/80 antibody (magnified as indicated). (C) Macrophage content was quantified by analyzing the fraction of F4/80-stained cells relative to total number of cells in epididymal adipose tissue from OPN $N^{+/+}$(black bars) and OPN $N^{-/-}$(white bars) mice fed a LFD or HFD ( $n=8$ /group). Values are expressed as mean \pm SEM. (D) Macrophage content was quantitatively assessed by real-time RT-PCR for CD68 mRNA expression in EWAT, the AF, and the SVF isolated from OPN $\mathrm{N}^{+/+}$(black bars) and $O P N^{-1-}$ (white bars) mice $(n=12 /$ group) fed a LFD or HFD for 25 weeks. Data are presented as relative CD68 mRNA expression normalized to TFIIB mRNA expression and are expressed as mean \pm SEM. ${ }^{*} P<0.05$, HFD compared with LFD; ${ }^{2} P<0.05, O P N^{-/-}$mice compared with $O P N^{+/+}$mice fed HFD.

(2), we further sought to investigate whether OPN deficiency and associated attenuation of macrophage accumulation in adipose tissue affects obesity-induced adipose tissue inflammation. As depicted in Figure 6, no significant differences in the expression of the inflammatory genes IL- 6 , TNF- $\alpha$, MCP-1, and iNOS were observed between lean $O P N^{+/+}$and $O P N^{-/-}$mice fed a LFD. However, HFD feeding and the development of DIO in $\mathrm{OPN}^{+/+}$mice markedly increased the expression of these inflammatory genes. Consistent with less ATM accumulation, IL-6, TNF- $\alpha$, and iNOS gene expression levels in adipose tissues isolated from obese $\mathrm{OPN}^{-/-}$ mice were significantly decreased. Similarly, MCP-1 expression levels revealed a trend to decrease in obese $O P N^{-/-}$mice, although this was not statistically significant. These data suggest that the obesity-associated increase of the inflammatory genes IL-6, TNF- $\alpha$, and iNOS in adipose tissue is OPN dependent.

We next determined the role of OPN for obesity-associated systemic inflammation and analyzed TNF- $\alpha$, IL- 6 , MCP-1, and plasminogen activator inhibitor 1 (PAI-1) plasma levels in $\mathrm{OPN}^{-/-}$and wild-type $\mathrm{OPN}^{+/+}$mice fed a LFD or HFD. TNF- $\alpha$ plasma levels were below detection limit in almost all samples analyzed using the mouse adipokine LincoPlex assay system (Figure 7A). Similarly, in lean mice fed a LFD, plasma levels of IL- 6 and MCP- 1 were below detection limit but increased to a detectable level in obese mice. PAI-1 plasma levels significantly increased in $\mathrm{OPN}^{+/+}$mice upon feeding a HFD. In $O P N^{-/-}$mice fed a HFD, however, plasma levels of IL-6, MCP-1, and PAI-1 were significantly lower compared 

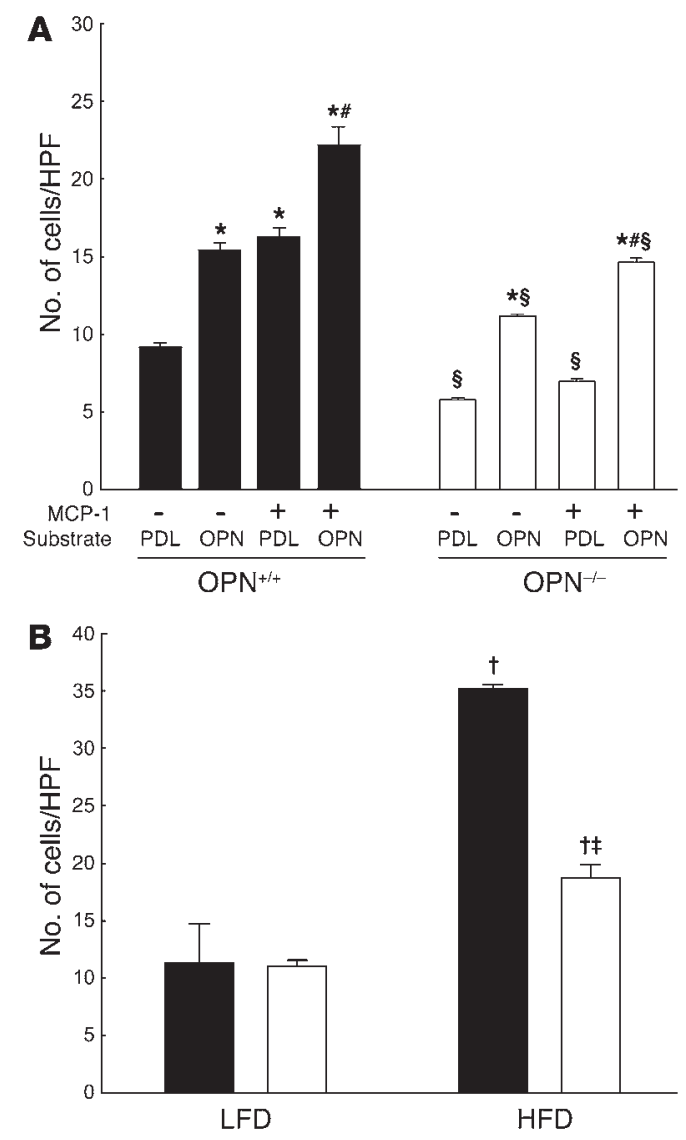

with those of wild-type $\mathrm{OPN}^{+/+}$mice. These data reveal that OPN modulates obesity-induced systemic inflammation by decreasing IL-6, MCP-1, and PAI-1 plasma levels.

Finally, we determined systemic levels of 3 adipokines that have been implicated in insulin sensitivity $(23,24)$. Adiponectin plasma levels decreased with the development of DIO and insulin resistance. Although there was a trend toward elevated adiponectin levels in LFD- and HFD-fed $O P N^{-/-}$mice compared with wild-type mice, this trend was not statistically significant. Compared with lean mice fed a LFD, serum leptin and resistin concentrations increased in obese HFD-fed mice and did not significantly differ between both genotypes (Figure 7B). Therefore, increased insulin

\section{Figure 5}

Chemotaxis of macrophages isolated from $O P N^{+/+}$and $O P N^{-/}$mice. (A) Peritoneal macrophages from $O P N^{+/+}$and $O P N^{-1-}$ mice were subjected to chemotaxis assays in modified Boyden chambers. Membranes of the transwell chambers were coated either with the substrate poly-Dlysine (PDL) as control or with recombinant OPN (5 ng/ml). Following attachment of the macrophages to the membrane, vehicle or MCP-1 $(50 \mathrm{ng} / \mathrm{ml})$ was added to the media in the lower chamber. Transwell migration was analyzed after 2 hours and expressed as cell numbers per HPF ( $\times 200)$. Experiments were repeated 4 times in triplicate. Data are expressed as mean \pm SEM. ${ }^{*} P<0.05$ compared with $\mathrm{PDL}$ alone; $\# P<0.05$ compared with OPN alone; $\$ P<0.05$ compared with $O P N^{+/+}$. (B) Stromal vascular cells were isolated from epididymal adipose tissues harvested from OPN $\mathrm{N}^{+/+}$(black bars) and $O P N^{-/-}$(white bars) mice fed a LFD or HFD for 25 weeks ( $n=6 /$ group). Cells were cultured in the bottom chambers, and peritoneal macrophages from wild-type mice were added to the insert. Migration was analyzed in triplicate after 2 hours as described in A. Data are expressed as mean \pm SEM. ${ }^{\dagger} P<0.05$, compared with LFD; $¥ P<0.05$, compared with $O P N^{+/+}$mice fed HFD.

sensitivity in obese $O P N^{-/-}$mice is likely the result of decreased ATM content, resulting in attenuated local and systemic inflammation rather than changes in insulin-sensitizing adipokine levels.

\section{Discussion}

OPN is a chemokine-like, extracellular matrix-associated protein involved in monocyte motility and the inflammatory immune response $(10,11)$. Since its initial cloning in 1986 (25), OPN has been associated with a remarkable range of pathologic responses (10); however, its role in obesity and metabolic disorders has not, to our knowledge, previously been investigated. In the present study, we demonstrate expression of OPN in adipose tissue and outline what we believe to be a previously unrecognized role for OPN to mediate obesity-associated ATM recruitment, adipose tissue inflammation, and resulting insulin resistance.

A host of adipose tissue-generated adipokines and cytokines have been identified that have emerged as an important source of systemic inflammation in obesity (2). We report here that OPN expression in adipose tissue increases with obesity and that plasma levels increase during the development of DIO OPN, suggesting a potential novel function of OPN for obesity-associated inflammatory and metabolic changes in adipose tissue. OPN plasma levels are elevated in various inflammatory diseases, including atherosclerosis (26), inflammatory bowel disease (27), granulomatous inflammatory diseases (28), rheumatoid arthritis (29), and mul-

\section{Figure 6}

Inflammatory gene expression in adipose tissues from $O P N^{+/+}$and $O P N^{-/-}$mice. $O P N^{+/+}$and $O P N^{-/-}$mice were fed a LFD or HFD for 25 weeks. mRNA expression levels of the indicated inflammatory genes were analyzed in EWATs isolated from OPN $\mathrm{N}^{+/+}$(black bars) and $\mathrm{OPN}^{-/-}$ (white bars) mice fed a LFD or OPN $N^{+/+}$(dark gray) and $O P N^{-/-}$mice (light gray) fed a HFD ( $n=6 /$ group). Data are presented as relative mRNA normalized to TFIIB mRNA and expressed as mean \pm SEM. ${ }^{*} P<0.05$, HFD compared with LFD; ${ }^{*} P<0.05, O P N^{-1-}$ mice compared with $\mathrm{OPN}^{+/+}$mice fed HFD.

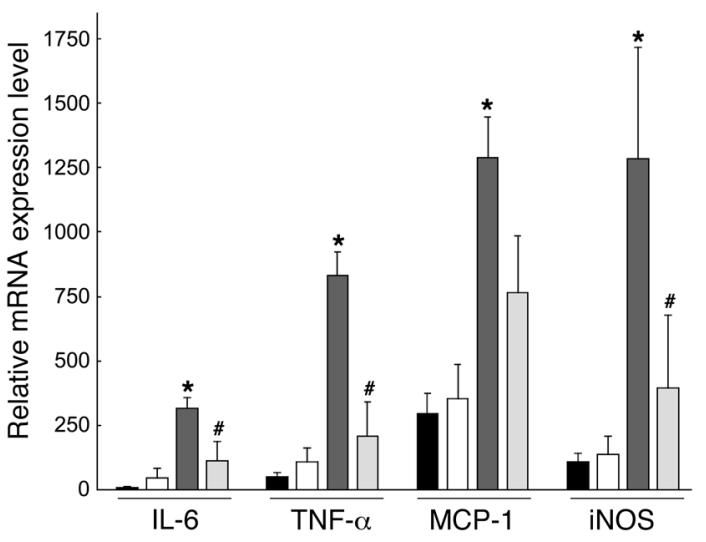



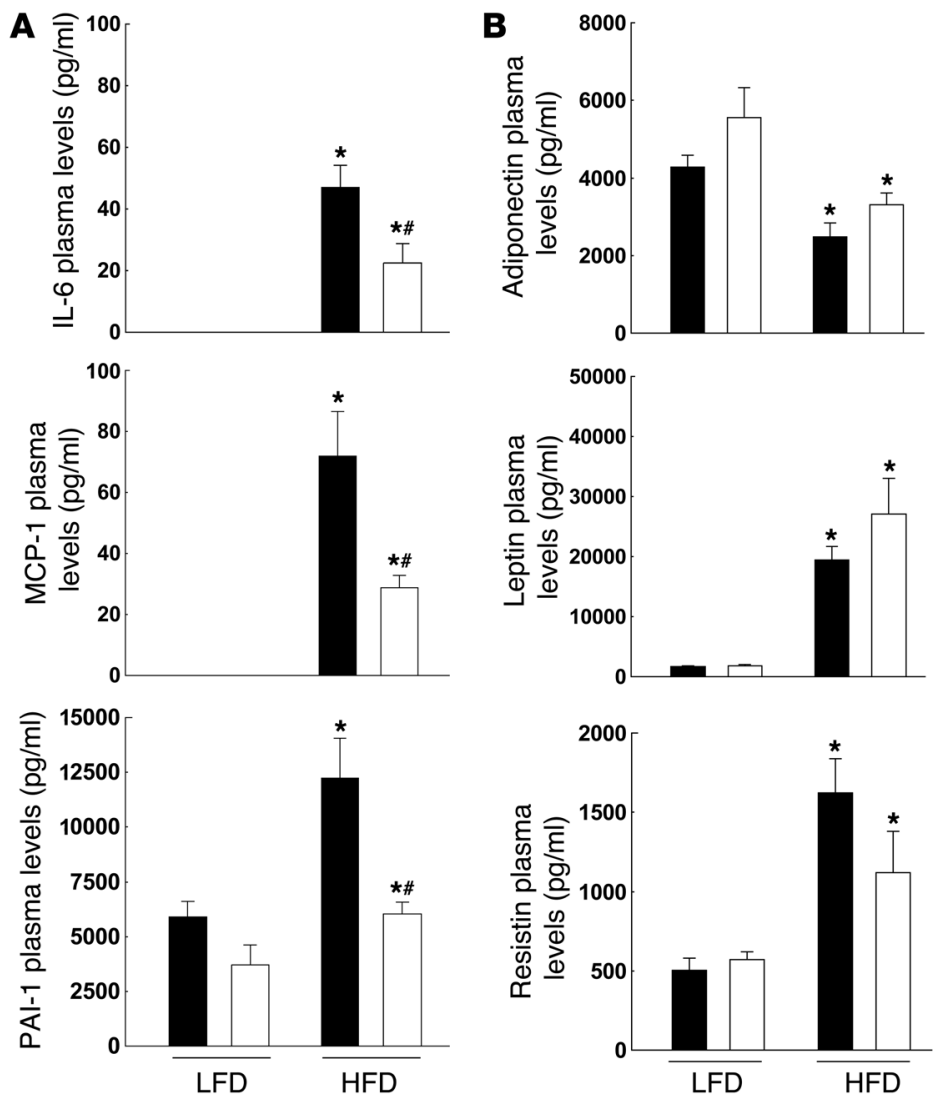

\section{Figure 7}

Plasma cytokine and adipokine levels in $\mathrm{OPN}^{+/+}$and $O P N^{-/-}$ mice. (A) IL-6, MCP-1, and PAI-1 plasma levels were analyzed in $\mathrm{OPN}^{+/+}$(black bars) and $\mathrm{OPN}^{-/-}$(white bars) mice $(n=10 /$ group) fed either a LFD or a HFD. (B) Plasma adiponectin, leptin, and resistin levels were analyzed in plasma obtained from mice described in A. Data are presented as mean \pm SEM. ${ }^{*} P<0.05$, compared with LFD; ${ }^{\#}<0.05$ compared with $O P N^{+/+}$ mice fed HFD.

decreased lean body mass in $O P N^{-/-}$mice. Our experiments employed NMR technology to provide measures of body fat mass, fat-free mass, and water content (37). Considering the limitation of this technique to distinguish different fat-free tissue masses including bone and muscle, the reason for the slightly decreased lean body mass in $O P N^{-/}$mice remains elusive. OPN deficiency renders mice less sensitive to bone resorption $(36,38)$. Thus this knowledge and the observation that bone density analyzed by micro-CT is not affected by OPN deficiency in unstressed mice (38) argues against a contribution of OPN deficiency in bone tissue to the observed decrease in lean body mass. To date no studies have determined whether OPN affects total body muscle mass. Therefore, it will be important for the determination of the mechanisms by which OPN deletion modulates lean body mass to characterize fat-free tissue masses in OPN-deficient mice and perform further studies using newly available techniques including NMR to analyze total body composition.

In bone marrow transplantation studies, Weisberg et al. have recently provided evidence that the majority of ATMs are derived from the circulation (3). The MCP-1/C-C motif

tiple sclerosis (30). OPN is rapidly expressed after cellular activation, and it is abundantly secreted by activated macrophages but not resting macrophages or monocytes $(31,32)$. Consistent with this notion, we observed that OPN expression in obese adipose tissue colocalized with macrophages and that OPN mRNA was highly expressed in macrophages isolated from the SVF. In contrast, we observed only negligible OPN mRNA in adipocytes and a decline in OPN transcript levels with differentiation of 3T3-L1 adipocytes. Although OPN is expressed in proliferating fibroblasts (33), these observations confirm earlier reports documenting decreased OPN mRNA levels in differentiated adipocytes as compared with preadipocytes (34). Interestingly, OPN has previously been characterized as a PPAR $\gamma$ target gene in macrophages, and overexpression of PPAR $\gamma$ or ligand treatment with a thiazolidinedione suppresses OPN transcription (17). PPAR $\gamma$ expression increases with differentiation of adipocytes, and when we overexpressed PPAR $\gamma$ in 3T3-L1 fibroblasts, thiazolidinedione treatment decreased OPN promoter activity. Similarly, overexpression of a constitutively active PPAR $\gamma$ mutant suppressed basal OPN promoter activity. Thus PPAR $\gamma$ mediated downregulation of OPN could provide a mechanism for the observed decline in OPN mRNA expression during the differentiation process of preadipocytes into mature adipocytes.

Using a model of DIO, our studies revealed that OPN has no effect on the development of obesity and does not affect food intake or energy metabolism. Consistent with several recent studies $(9,35,36)$, total body weights were similar in wild-type and OPN-deficient mice. However, in previous studies body composition was not analyzed, and an unexpected but interesting and consistent observation in both lean and obese animals was a slightly chemokine receptor 2 (MCP-1/CCR2) axis is well established to regulate macrophage recruitment to sites of inflammation (39, 40 ), and MCP-1 is secreted from adipocytes and plasma levels are increased in obesity (41). In addition, MCP-1 levels secreted from adipocytes correlate with adipocyte size (42), indicating that the MCP-1/CCR2 cascade is likely among the earliest mechanisms involved in the recruitment of monocytes to adipose tissue. This is further supported by recent studies demonstrating that adipocytespecific MCP-1 overexpression results in enhanced macrophage infiltration of adipose tissue $(43,44)$, while MCP-1 or CCR2 deficiency decreases macrophage content in obese adipose tissue (19, 43). However, in both models obesity-induced macrophage accumulation in adipose tissue is not normalized and macrophages remain accumulating in the adipose tissue, suggesting that additional mechanisms are involved in this process. In this study, we demonstrate decreased macrophage content in obese adipose tissue from $O P N^{-/-}$mice in the absence of any differences in total fat mass, thus providing an additional mechanism by which macrophages infiltrate adipose tissue. This requirement of OPN for macrophage recruitment to adipose tissue is consistent with our data examining the role of OPN for macrophage chemotaxis. In wild-type macrophages OPN amplifies macrophage migration and exerts additive effects on chemotaxis in the presence of MCP-1. In contrast, OPN-deficient macrophages are hypomotile and less responsive to MCP-1, a phenotype that exogenous OPN is unable to completely correct. These observations suggest that OPN augments the MCP-1 response and functions primarily in an autocrine mechanism to promote macrophage chemotaxis. This concept is in agreement with recent studies demonstrating that endogenous OPN expres- 
sion in macrophages is important to maintain macrophage function, including chemotaxis, differentiation, and inflammation (13). Moreover, intracellular OPN forms a complex with the CD44 receptor and ezrin/radixin/moesin proteins at the cell membrane of cell processes (45) that is required for cell fusion and chemotaxis of macrophages (22). Finally, it is well recognized that $O P N^{-1-}$ mice have defective granulomatous responses $(8,46)$ that likely involve abnormal macrophage function.

The observation that OPN is primarily expressed by macrophages in obese adipose tissue combined with the important autocrine role of OPN in macrophage function suggest a model in which endogenous OPN amplifies macrophage recruitment through the MCP-1/CCR2 cascade in the early stages of obesity. Our ex vivo experiments demonstrate increased macrophage chemotaxis toward the SVF isolated from obese mice, indicating that the continued recruitment of macrophages within the SVF may further exacerbate macrophage infiltration. In contrast, migration toward the SVF isolated from obese $\mathrm{OPN}^{-/-}$mice was substantially decreased. Our findings that macrophage content in the SVF from $O P N^{-/-}$mice is considerably less compared with that from $\mathrm{OPN}^{+/+}$mice may indicate that fewer macrophages accumulating during the course of obesity ultimately secrete fewer migratory signals, which would likely contribute to decreased macrophage recruitment during later stages of obesity. In concert, these studies support an important role for OPN to promote macrophage infiltration into obese adipose tissue. However, confirming the contribution macrophage-dervied OPN to adipose tissue inflammation in vivo requires studies that will depend on the specific deletion of OPN in macrophages using either bone marrow transplantation approaches or conditional OPN deletion strategies.

Concomitant with the attenuated macrophage content in obese adipose tissue from $\mathrm{OPN}^{-/-}$mice, we documented decreased inflammatory gene expression in adipose tissue and decreased systemic levels of the proinflammatory cytokines IL-6, MCP-1, and PAI-1. MCP-1- and CCR2-deficient mice develop less obesity-induced insulin resistance $(19,43)$, and the current understanding of the role of ATMs and adipose tissue inflammation in obesity-induced insulin resistance would suggest that prevention of macrophage accumulation in adipose tissue preserves insulin sensitivity. In further support of this concept, we demonstrate that decreased ATM accumulation in OPN-deficient mice is associated with increased insulin sensitivity: $O P N^{-/-}$mice developed less obesity-associated hyperinsulinemia, cleared glucose more rapidly following an intraperitoneal glucose challenge, and exhibited an enhanced insulin response after an intraperitoneal injection of insulin. Importantly, these effects of OPN deficiency were observed despite the same level of obesity in $\mathrm{OPN}^{-/-}$and wild-type mice. Increased insulin sensitivity associated with OPN deficiency was unlikely a result of altered adipokine secretion, since plasma levels of 3 adipokines implicated in insulin resistance (adiponectin, resistin, and leptin) were not significantly different in $O P N^{-/-}$mice. Based on the strong recent evidence that ATMs are both necessary and sufficient for the development of obesity-associated insulin resistance $(4,19$, 43), the observed decrease in ATM content in obese $\mathrm{OPN}^{-/-}$mice provides a likely mechanism for the increased insulin sensitivity in these mice. Macrophages present in adipose tissue directly interfere with insulin signaling and insulin-stimulated glucose uptake in adipocytes by decreasing GLUT4 and insulin receptor substrate 1 (IRS-1) expression, leading to a decrease in Akt phosphorylation and impaired insulin-stimulated GLUT4 translocation to the plas- ma membrane (47). Interestingly, blocking TNF- $\alpha$, which is well established to contribute to insulin resistance (48), prevents macrophage-induced alterations in adipocyte insulin signaling (47). These observations confirm important cross-talk between ATMs and adipocytes in mediating insulin resistance and indicate that ATMs affect insulin signaling by perpetuating inflammatory pathways in adipocytes. While the concept of decreased ATM content in $O P N^{-/-}$mice as a mechanism for improved insulin sensitivity is intriguing and supported by these studies, we cannot exclude that OPN may also affect hepatic insulin sensitivity. OPN deficiency has previously been associated with decreased hepatic fibrosis in experimental nonalcoholic steatohepatitis (49), and since hepatic OPN expression increases during nonalcoholic steatohepatitis in obese mice (50), OPN deficiency may also modulate obesityinduced hepatic insulin resistance.

In summary, in the present study we characterize OPN, a versatile mediator of macrophage motility involved in cell-mediated inflammation $(9-11,22,51)$, as a novel cytokine expressed by ATMs and secreted during DIO. OPN expression is required for macrophage recruitment into adipose tissue and for obesity-associated adipose tissue and systemic inflammation. Finally, we demonstrate that decreased adipose tissue inflammation in $\mathrm{OPN}^{-/-}$mice is associated with improved obesity-associated insulin resistance without significantly altering body mass. These data therefore identify OPN as a previously unappreciated link between obesity, adipose tissue inflammation, and insulin resistance.

\section{Methods}

Animals and animal care. $\mathrm{OPN}^{-/-}$mice on a BlackSwiss background, kindly provided by C.M. Giachelli (University of Washington, Seattle, Washington, USA) and L. Liaw (Maine Medical Center Research Institute, Scarborough, Maine, USA), were used as previously described (9). C57BL/ 6 mice were obtained from The Jackson Laboratory at 6-8 weeks of age. All mice were housed in Plexiglas ventilated cages ( 4 animals/cage) within a pathogen-free barrier facility that maintained a 12 -hour light/12-hour dark cycle. Mice had access to autoclaved water and pellet food ad libitum. Prior to 8 weeks of age, all mice were fed a standard rodent chow diet containing approximately $5 \% \mathrm{kcal}$ fat (Diet 7012; Harlan Teklad). At 8 weeks of age, male $\mathrm{OPN}^{-/-}$and littermate wild-type $\mathrm{OPN}^{+/+}$mice were fed diets containing either 10\% kcal (LFD) or 60\% kcal (HFD) from fat (D12450B and D12492; Research Diets Inc.) for 25 weeks. Weight gain during DIO was monitored by weighing mice weekly. All animal studies were in compliance with the Division of Laboratory Animal Research Guide for Care and Use of Laboratory Animals and were performed with the approval of the University of Kentucky Institutional Animal Care and Use Committee.

Analysis of body composition, energy expenditure, food intake, and locomotor activity. Mice were analyzed for body composition by quantitative NMR (37) at the University of Cincinnati Mouse Metabolic Phenotyping Center before and after being fed a HFD. Food intake, energy expenditure, RQ, and locomotor activity were analyzed using a calorimetry system (LabMaster; TSE Systems). Mice were placed in the calorimetry system and adapted for 48 hours, and cumulative recordings were collected over the following 48 hours.

Isolation of adipose tissues. Mice fed a LFD or HFD were sacrificed by $\mathrm{CO}_{2}$ asphyxiation, and EWAT pads were removed. For adipose tissue fractionation into the SVF or AF, epididymal adipose tissue pads were minced, placed in HEPES-buffered DMEM (Invitrogen) supplemented with $10 \mathrm{mg} /$ $\mathrm{ml}$ fatty acid-poor BSA (FAP-BSA; Sigma-Aldrich), and centrifuged at $500 \mathrm{~g}$ for 10 minutes. Following centrifugation, samples were incubated with $840 \mathrm{U} / \mathrm{g}$ collagenase type I (Worthington Chemicals) at $37^{\circ} \mathrm{C}$ with gentle agitation for 1 hour. The suspension was then passed through a sterile 
250- $\mu \mathrm{m}$ nylon mesh (Sefar America Inc.) and centrifuged at $500 \mathrm{~g}$ for 10 minutes. Pelleted cells were collected as the SVF, and the floating cells were collected as the AF. The AF was subsequently further digested for 1 hour, washed twice with DMEM, and subjected to a second centrifugation step as described above until there were no further cell or debris pellets. The SVF was resuspended in erythrocyte lysis buffer, incubated at room temperature for 3 minutes, and centrifuged at $500 \mathrm{~g}$ for 5 minutes. For isolation of mRNA, the erythrocyte-depleted SVF and the AF were resuspended in TRIzOL reagent (Invitrogen). Separation of ATMs and endothelial cells from SVF isolates was performed by magnetic immunoaffinity isolation using anti-CD11b and anti-CD31 antibodies conjugated to magnetic beads (MACS Cell Separation System; Miltenyi Biotec), respectively. Following isolation of ATMs and endothelial cells from the SVF using positive selection columns (MS columns; Miltenyi Biotec), the remaining cells were eluted as the preadipocyte fraction. For the analysis of OPN mRNA expression levels, eluted cell isolates were resuspended in TRIzOL reagent.

Metabolic measurements. Blood samples were obtained from wild-type $\mathrm{OPN}^{+/+}$and $\mathrm{OPN}^{-/-}$mice after feeding a LFD or HFD for 25 weeks. The blood was collected after an overnight fast from the retroorbital plexus or from the abdominal vena cava at sacrifice. Plasma glucose, cholesterol, and triglyceride concentrations were determined using commercially available enzymatic assay kits (Wako Chemicals). Plasma levels for insulin, leptin, resistin, IL-6, MCP-1, TNF- $\alpha$, and PAI- 1 were analyzed at Linco Diagnostics using the mouse adipokine assay kit. Adiponectin plasma levels were analyzed using a mouse adiponectin ELISA kit (Linco Diagnostics). OPN plasma levels were analyzed using a commercially available mouse OPN EIA Kit (Assay Designs). Insulin sensitivity and glucose tolerance were analyzed in LFD- and HFD-fed animals after an overnight fasting period (14 hours). Following an intraperitoneal bolus injection of recombinant human regular insulin (1 U/kg body weight) (Novolin R; Novo Nordisk Inc.), blood glucose concentrations were measured using a Freestyle Flash Glucometer (Abbott Laboratories) before and 30, 60, 90, and 120 minutes after injection. Glucose tolerance tests were performed following an intraperitoneal injection of dextrose dissolved in water $(1 \mathrm{~g} / \mathrm{kg}$ body weight). Blood glucose levels were analyzed before and 30, 60, 90, 120, 150, and 180 minutes following injection.

Immunohistochemistry. For histological analysis of OPN expression in adipose tissue, EWAT tissues were isolated from C57BL/6 mice fed a HFD for 3 months. Immunohistochemistry on paraffin-embedded tissues was performed as described in ref. 9 using antibodies for OPN (dilution of $2.5 \mu \mathrm{g} / \mathrm{ml}$, catalog no. AF808; R\&D Systems) and F4/80 (dilution of 1:50, catalog no. ab6640; Abcam).

Analysis of macrophage content in epididymal adipose tissues was performed on tissue pads isolated from $O P N^{-/-}$and $O P N^{+/+}$mice fed either a LFD or HFD for 25 weeks. At sacrifice, EWAT pads were embedded in OCT compound (Tissue-Tek; Miles Inc.) and snap-frozen. Transverse cryosections $(10 \mu \mathrm{m}$ thick) were collected at $100 \mu \mathrm{M}$ intervals and fixed in cold acetone. Macrophages were detected using a rabbit anti-mouse macrophage anti-serum at a dilution of 1:10,000 (catalog no. AIAD31240; Accurate Chemical \& Scientific Corp.) (9) or an F4/80 antibody at a dilution of 1:50 (catalog no. ab-6640; Abcam). Two observers blinded to the experimental design performed quantification of ATM content in 5 sections obtained from each adipose tissue pad. The total number of nuclei and the number of nuclei of F4/80-expressing cells were counted in 10 high-power fields (original magnification, $\times 200$ ) of each section using the image analysis software ImagePro Plus (version 4.1; Media Cybernetics Inc.). The ratio of nuclei in F4/80-positive cells and total number of nuclei in each sample provided the fraction of ATMs for each section analyzed.

Cell culture and transient transfection. We maintained 3T3-L1 fibroblasts (ATCC) in DMEM supplemented with 10\% fetal bovine serum. Adipogene- sis was induced using standard protocols for treatment of cells with insulin $(0.1 \mu \mathrm{M})$, dexamethasone $(1 \mu \mathrm{M})$, and isobutylmethylxanthine $(0.5 \mathrm{mM})$. mRNA was isolated at various time points for the analysis of OPN and $\operatorname{PPAR} \gamma$ mRNA during the differentiation process. The OPN promoter construct, wild-type PPAR $\gamma$ expression vector, and constitutively active PPAR $\gamma$ expression vector have previously been described (52-54). We seeded 3T3L1 fibroblasts on 6-well plates and transiently cotransfected them with $1 \mu \mathrm{g}$ DNA of the OPN promoter construct and $500 \mathrm{ng}$ empty control vector, wild-type PPAR $\gamma 2$ expression vector, or constitutively active PPAR $\gamma$ expression vector using Lipofectamine 2000 (Invitrogen). Transfection efficiency was normalized to renilla luciferase activities generated by cotransfection with $10 \mathrm{ng} /$ well pRL-CMV (Promega). At 8 hours after transfection, cells were treated with vehicle (DMSO) or $10 \mu \mathrm{M}$ pioglitazone (kindly provided by Takeda Pharmaceuticals North America). Luciferase activity was assayed 24 hours after stimulation as described in ref. 54. All experiments were repeated at least 3 times and performed in triplicate.

Chemotaxis assays. Chemotaxis assays were performed using murine peritoneal macrophages in a modified Boyden chamber transwell migration assay (24-well plates, $8 \mu \mathrm{m}$ pore size; catalog no. 3422; Costar Inc.) as described previously (9). Peritoneal macrophages from 8-week-old BlackSwiss wild-type mice were collected 3 days after injection of $1 \mathrm{ml}$ of $1 \%$ thioglycollate into the peritoneal cavity. Microporous membranes of the transwell inserts were coated with poly-D lysine (Sigma-Aldrich) or OPN $5 \mathrm{ng} / \mathrm{ml}$ (R\&D Systems) and air dried for 2 hours. Macrophages $\left(2 \times 10^{4}\right)$ were plated to each insert in $100 \mu \mathrm{l}$ DMEM containing $0.4 \% \mathrm{FBS}$, and 600 $\mu \mathrm{l}$ medium was added to each lower well. The cells were allowed to attach to the membranes for 2 hours at $37^{\circ} \mathrm{C}$ in a humidified $\mathrm{CO}_{2}$ incubator, and then the chemoattractant MCP-1 ( $50 \mathrm{ng} / \mathrm{ml}$; R\&D Systems) or vehicle was added to the lower wells. In ex vivo experiments, stromal vascular cells from EWAT pads were isolated from $O P N^{+/+}$and $O P N^{-/}$mice fed a HFD or LFD for 25 weeks. Cells were cultured in the bottom chambers of the culture plate, and after 2 days wild-type peritoneal macrophages were added to the insert. In both experiments cells were allowed to migrate for 2 hours and fixed with $2 \%$ paraformaldehyde. The non-migrating cell fraction on the top surface of the membrane was removed with cotton swabs, while the migrated cells were stained with hematoxylin and quantitated in triplicate sets of wells. Five randomly chosen high-power fields were counted per membrane. Experiments were performed at least 3 times with different preparations of macrophages and stromal vascular cells.

Reverse transcription and quantitative real-time RT-PCR. OPN, CD68, IL-6, TNF- $\alpha$, MCP- 1 , and iNOS mRNA expression was measured by quantitative real-time RT-PCR as indicated in Figure 1, B and C, Figure 4D, and Figure 6. Total mRNA of these tissues was isolated using TRIzOL (Invitrogen) and reverse transcribed into cDNA as previously described (9). PCR reactions were performed using an iCycler (Bio-Rad) and SYBR Green I system (Bio-Rad). Each sample was analyzed in triplicate and normalized to values for TFIIB mRNA expression. Mouse primer sequences used were as follows: OPN, 5'-TCCCTCGATGTCATCCCTGT-3' (forward), 5' -CCCTTTCCGTTGTTGTCCTG-3' (reverse); CD68, 5'-CAAGGTCCAGGGAGGTTGTG-3' (forward), 5'-CCAAAGGTAAGCTGTCCATAAGGA-3' (reverse); IL-6, 5'-CTGCAAGAGACTTCCATCCAGTT-3' (forward), 5'-GAAGTAGGGAAGGCCGTGG-3' (reverse); TNF- $\alpha, 5^{\prime}$-AGCCGATGGGTTGTACCT-3' (forward), 5'-TGAGTTGGTCCCCCTTCT-3' (reverse); MCP-1, 5' -CAGCCAGATGCAGTTAACGC3' (forward), 5'-GCCTACTCATTGGGATCATCTTG-3' (reverse); iNOS, 5'-GCGCTGGGCTGTACAAA-3' (forward), 5'-AGCGTTTCGGGATCTGAAT-3' (reverse); PPAR $\gamma, 5^{\prime}$-TGCTGTTATGGGTGAAACTCTG-3' (forward), 5' - CTGTGTCAACCATGGTAATTTCTT-3' (reverse); and TFIIB, 5'-CTCTCCCAAGAGTCACATGTCC-3' (forward), 5' -CAATAACTCGGTCCCCTACAAC-3' (reverse). 
Statistics. Statistical analysis for pairwise comparisons between different genotypes or diets was performed using the 2-tailed Student's $t$ test. To analyze the interaction between the genotype and the diet, 2-way ANOVA was performed. Analyses were performed using Statistica 7 (Statsoft Inc.).

\section{Acknowledgments}

These studies were supported in part by grants from the NIH (HL084611), the American Diabetes Association (Research Award 1-06-RA-17), the American Heart Association (Scientist Development Grant $0435239 \mathrm{~N}$ ), and by institutional funds from the University of Kentucky College of Medicine to D. Bruemmer. This work was further supported in part by the Cincinnati Mouse Metabolic Phenotyping Center ID DK59630 to M.H. Tschöp. T.
Nomiyama and F. Gizard were supported by postdoctoral fellowships from the American Heart Association Great Rivers Affiliate (0725620B and 0725313B, respectively).

Received for publication February 28, 2007, and accepted in revised form July 25, 2007.

Address correspondence to: Dennis Bruemmer, University of Kentucky College of Medicine, Department of Internal Medicine, Division of Endocrinology and Molecular Medicine, Wethington Health Sciences Building, Room 575, 900 South Limestone Street, Lexington, Kentucky 40536-0200, USA. Phone: (859) 323-4933, ext. 81418; Fax: (859) 257-3646; E-mail: dennis.bruemmer@uky.edu.
1. Van Gaal, L.F., Mertens, I.L., and De Block, C.E. 2006. Mechanisms linking obesity with cardiovascular disease. Nature. 444:875-880.

2. Hotamisligil, G.S. 2006. Inflammation and metabolic disorders. Nature. 444:860-867.

3. Weisberg, S.P., et al. 2003. Obesity is associated with macrophage accumulation in adipose tissue. J. Clin. Invest. 112:1796-1808. doi:10.1172/ JCI200319246.

4. Xu, H., et al. 2003. Chronic inflammation in fat plays a crucial role in the development of obesity-related insulin resistance. J. Clin. Invest. 112:1821-1830. doi:10.1172/JCI200319451.

5. Wellen, K.E., and Hotamisligil, G.S. 2003. Obesity-induced inflammatory changes in adipose tissue. J. Clin. Invest. 112:1785-1788. doi:10.1172/ JCI200320514.

6. Nau, G.J., et al. 1997. A chemoattractant cytokine associated with granulomas in tuberculosis and silicosis. Proc. Natl. Acad. Sci. U. S. A. 94:6414-6419.

7. Giachelli, C.M., Lombardi, D., Johnson, R.J., Murry, C.E., and Almeida, M. 1998. Evidence for a role of osteopontin in macrophage infiltration in response to pathological stimuli in vivo. Am.J. Pathol. 152:353-358.

8. Ashkar, S., et al. 2000. Eta-1 (osteopontin): an early component of type-1 (cell-mediated) immunity. Science. 287:860-864.

9. Bruemmer, D., et al. 2003. Angiotensin II-accelerated atherosclerosis and aneurysm formation is attenuated in osteopontin-deficient mice. J. Clin. Invest. 112:1318-1331. doi:10.1172/JCI200318141.

10. Denhardt, D.T., Giachelli, C.M., and Rittling, S.R. 2001. Role of osteopontin in cellular signaling and toxicant injury. Annu. Rev. Pharmacol. Toxicol. 41:723-749.

11. Denhardt, D.T., Noda, M., O’Regan, A.W., Pavlin, D., and Berman, J.S. 2001. Osteopontin as a means to cope with environmental insults: regulation of inflammation, tissue remodeling, and cell survival. J. Clin. Invest. 107:1055-1061.

12. Reinholt, F., Hultenby, K., Oldberg, A., and Heinegard, D. 1990. Osteopontin - a possible anchor of osteoclast to bone. Proc. Natl. Acad. Sci. U. S. A. 87:4473-4475.

13. Nystrom, T., Duner, P., and Hultgardh-Nilsson, A. 2007. A constitutive endogenous osteopontin production is important for macrophage function and differentiation. Exp. Cell Res. 313:1149-1160.

14. Murry, C.E., Giachelli, C.M., Schwartz, S.M., and Vracko, R. 1994. Macrophages express osteopontin during repair of myocardial necrosis. Am. J. Pathol. 145:1450-1462.

15. Matsui, Y., et al. 2003. Osteopontin deficiency attenuates atherosclerosis in female apolipoprotein E-deficient mice. Arterioscler. Thromb. Vasc. Biol. 23:1029-1034.

16. Lumeng, C.N., Bodzin, J.L., and Saltiel, A.R. 2007. Obesity induces a phenotypic switch in adipose tissue macrophage polarization. J. Clin. Invest.
117:175-184. doi:10.1172/JCI29881

17. Oyama, Y., Akuzawa, N., Nagai, R., and Kurabayashi, M. 2002. PPAR \{gamma\} Ligand inhibits osteopontin gene expression through interference with binding of nuclear factors to A/T-rich sequence in THP-1 cells. Circ. Res. 90:348-355.

18. Micklem, K., et al. 1989. A human macrophageassociated antigen (CD68) detected by six different monoclonal antibodies. Br. J. Haematol. 73:6-11.

19. Weisberg, S.P., et al. 2006. CCR2 modulates inflammatory and metabolic effects of high-fat feeding. J. Clin. Invest. 116:115-124. doi:10.1172/JCI24335.

20. Hirsch, S., Austyn, J.M., and Gordon, S. 1981. Expression of the macrophage-specific antigen F4/80 during differentiation of mouse bone marrow cells in culture. J. Exp. Med. 154:713-725.

21. Surwit, R.S., Kuhn, C.M., Cochrane, C., McCubbin, J.A., and Feinglos, M.N. 1988. Diet-induced type II diabetes in C57BL/6J mice. Diabetes. 37:1163-1167.

22. Zhu, B., et al. 2004. Osteopontin modulates CD44dependent chemotaxis of peritoneal macrophages through G-protein-coupled receptors: evidence of a role for an intracellular form of osteopontin. J. Cell. Physiol. 198:155-167.

23. Trujillo, M.E., and Scherer, P.E. 2006. Adipose tissue-derived factors: impact on health and disease. Endocr. Rev. 27:762-778.

24. Kershaw, E.E., and Flier, J.S. 2004. Adipose tissue as an endocrine organ. J. Clin. Endocrinol. Metab. 89:2548-2556.

25. Oldberg, A., Franzen, A., and Heinegard, D. 1986. Cloning and sequence analysis of rat bone sialoprotein (osteopontin) cDNA reveals an Arg-Gly-Asp cell-binding sequence. Proc. Natl. Acad. Sci. U. S. A. 83:8819-8823.

26. Ohmori, R., et al. 2003. Plasma osteopontin levels are associated with the presence and extent of coronary artery disease. Atherosclerosis. 170:333-337.

27. Sato, T., et al. 2005. Osteopontin/Eta-1 upregulated in Crohn's disease regulates the Th1 immune response. Gut. 54:1254-1262.

28. Koguchi, Y., et al. 2003. High plasma osteopontin level and its relationship with interleukin-12-mediated type $1 \mathrm{~T}$ helper cell response in tuberculosis. Am. J. Respir. Crit. Care Med. 167:1355-1359.

29. Gattorno, M., et al. 2004. Synovial expression of osteopontin correlates with angiogenesis in juvenile idiopathic arthritis. Rheumatology. 43:1091-1096.

30. Vogt, M.H., Lopatinskaya, L., Smits, M., Polman, C.H., and Nagelkerken, L. 2003. Elevated osteopontin levels in active relapsing-remitting multiple sclerosis. Ann. Neurol. 53:819-822.

31. Miyazaki, Y., et al. 1990. The mouse osteopontin gene. Expression in monocytic lineages and complete nucleotide sequence. J. Biol. Chem. 265:14432-14438.

32. Somerman, M.J., Berry, J.E., Khalkhali-Ellis, Z., Osdoby, P., and Simpson, R.U. 1995. Enhanced expression of [alpha]V integrin subunit and osteopontin during differentiation of HL-60 cells along the monocytic pathway. Exp. Cell Res. 216:335-341.
33. Senger, D.R., Asch, B.B., Smith, B.D., Perruzzi, C.A., and Dvorak, H.F. 1983. A secreted phosphoprotein marker for neoplastic transformation of both epithelial and fibroblastic cells. Nature. 302:714-715.

34. Dorheim, M.A., et al. 1993. Osteoblastic gene expression during adipogenesis in hematopoietic supporting murine bone marrow stromal cells. J. Cell. Physiol. 154:317-328.

35. Koyama, Y., et al. 2006. Osteopontin deficiency suppresses high phosphate load-induced bone loss via specific modulation of osteoclasts. Endocrinology. 147:3040-3049.

36. Yoshitake, H., Rittling, S.R., Denhardt, D.T., and Noda, M. 1999. Osteopontin-deficient mice are resistant to ovariectomy-induced bone resorption. Proc. Natl. Acad. Sci. U. S. A. 96:8156-8160.

37. Tinsley, F.C., Taicher, G.Z., and Heiman, M.L. 2004. Evaluation of a quantitative magnetic resonance method for mouse whole body composition analysis. Obes. Res. 12:150-160.

38. Ishijima, M., et al. 2001. Enhancement of osteoclastic bone resorption and suppression of osteoblastic bone formation in response to reduced mechanical stress do not occur in the absence of osteopontin. J. Exp. Med. 193:399-404.

39. Boring, L., et al. 1997. Impaired monocyte migration and reduced type 1 (Th1) cytokine responses in C-C chemokine receptor 2 knockout mice. J. Clin. Invest. 100:2552-2561.

40. Rollins, B.J., Walz, A., and Baggiolini, M. 1991. Recombinant human MCP-1/JE induces chemotaxis, calcium flux, and the respiratory burst in human monocytes. Blood. 78:1112-1116.

41. Sartipy, P., and Loskutoff, D.J. 2003. Monocyte chemoattractant protein 1 in obesity and insulin resistance. Proc. Natl. Acad. Sci. U. S. A. 100:7265-7270.

42. Skurk, T., Alberti-Huber, C., Herder, C., and Hauner, H. 2006. Relationship between adipocyte size and adipokine expression and secretion. J. Clin. Endocrinol. Metab. 92:1023-1033.

43. Kanda, H., et al. 2006. MCP-1 contributes to macrophage infiltration into adipose tissue, insulin resistance, and hepatic steatosis in obesity. J. Clin. Invest. 116:1494-1505. doi:10.1172/JCI26498.

44. Kamei, N., et al. 2006. Overexpression of monocyte chemoattractant protein-1 in adipose tissues causes macrophage recruitment and insulin resistance. J. Biol. Chem. 281:26602-26614.

45. Zohar, R., et al. 2000. Intracellular osteopontin is an integral component of the CD44-ERM complex involved in cell migration. J. Cell. Physiol. 184:118-130.

46. Nau, G.J., et al. 1999. Attenuated host resistance against Mycobacterium bovis BCG infection in mice lacking osteopontin. Infect. Immun. 67:4223-4230.

47. Lumeng, C.N., Deyoung, S.M., and Saltiel, A.R. 2007. Macrophages block insulin action in adipocytes by altering expression of signaling and glucose transport proteins. Am. J. Physiol. Endocrinol. Metab. 292:E166-E174.

48. Hotamisligil, G.S., Shargill, N.S., and Spiegelman, 
B.M. 1993. Adipose expression of tumor necrosis factor-alpha: direct role in obesity-linked insulin resistance. Science. 259:87-91.

49. Sahai, A., Malladi, P., Melin-Aldana, H., Green, R.M., and Whitington, P.F. 2004. Upregulation of osteopontin expression is involved in the development of nonalcoholic steatohepatitis in a dietary murine model. Am. J. Physiol. Gastrointest. Liver Physiol. 287:G264-G273.

50. Sahai, A., et al. 2004. Obese and diabetic db/db mice develop marked liver fibrosis in a model of nonalcoholic steatohepatitis: role of short-form leptin receptors and osteopontin. Am. J. Physiol. Gastrointest. Liver Physiol. 287:G1035-G1043.

51. Weber, G.F., et al. 2002. Phosphorylation-dependent interaction of osteopontin with its receptors regulates macrophage migration and activation. J. Leukoc. Biol. 72:752-761.

52. Ogawa, D., et al. 2005. Liver $\mathrm{x}$ receptor agonists inhibit cytokine-induced osteopontin expression in macrophages through interference with activator protein-1 signaling pathways. Circ. Res. 96:e59-e67. 53. Schupp, M., Janke, J., Clasen, R., Unger, T., and Kintscher, U. 2004. Angiotensin type 1 receptor blockers induce peroxisome proliferator-activated receptor\{gamma\} activity. Circulation. 109:2054-2057.

54. Bruemmer, D., et al. 2003. Peroxisome proliferatoractivated receptor gamma inhibits expression of minichromosome maintenance proteins in vascular smooth muscle cells. Mol. Endocrinol. 17:1005-1018. 